\title{
ASYMPTOTIC ANALYSIS OF THE NON-STATIONARY CROSS-FLOW VORTICES ON A ROTATING DISK WITH TEMPERATURE-DEPENDENT VISCOSITY
}

\author{
H. A. JASMINE \\ Department of Mathematics, University of Rajshahi, Rajshahi, Bangladesh \\ Email hjasmine@,hotmail.com
}

Received 08.05.07 Accepted 19.04.08

\begin{abstract}
Asymptotic analysis of the non-stationary cross-flow disturbances of Von-kármán rotation disk flow with a temperature dependence viscosity is investigated. The linear eigenrelations are derived for various values of the parameter which controls the temperature dependence of viscosity with more than one critical layer. It has been fund that there is a cut-off value at an angle that lies between $10.3^{\circ}$ and $57.4^{0}$, so that solution exist only for this range.
\end{abstract}

\section{INTRODUCTION}

Rotating disk flow has been at the centre of a large number of theoretical and experimental studies in recent years. In this flow temperature changes, viscosity can also undergo a significant change. To predict the behaviour of a flow properly, it is therefore necessary to consider viscosity variation for incompressible fluids. When the viscosity variation on temperature is taken into account, Gary et al. (1), and Mehta \& Sood [2] demonstrated substantial change in flow characteristics compared to the constant viscosity assumption. Kafoussias \& Williams [3], Kafoussias \& Rees [4], and Keller [5] studied the effects of temperature dependent viscosity on mixed and natural convection flows by considering the viscosity to vary as linear function of temperature or proportional to a liner function of temperature.

Hossain et al. [6] investigated the flow and heat transfer along a uniformly heated and impulsively rotating disk in a stationary fluid subjected to a trans-verse magnetic field with temperature dependent viscosity. The flow considered is laminar with viscosity $\mu^{\prime}=$ $\mu \infty /[1+\in(T-T \infty) /(T \omega-T \infty)]$ and it is shown that heat transfer and surface friction are affected by the flow in the viscous sub-layer close to the disk surface, Using a finite difference method, Hossain et al. [7] explored the effect of a temperature dependent viscosity on natural convection flow of a viscous incompressible fluid from a vertical wavy surface. The influence of temperature dependent viscosity on the flow along a channel with porous wall was recently analyzed by Ferro \& Gnavi [8]. Absolute and convective instabilities in the incompressible boundary layer on a rotationg disk with temperature-dependent viscosity has been solved numerically by jasmine \& Gajjar [9].

Bassom \& Gajjar [10] have investigated the upper-branch neutral stability of three dimensional disturbances imposed on a three-dimensional boundary layer profile and non-stationary cross-flow vortices for constant viscosity. In this section our objective is to 
extend Bassom \& Gajjar [10] formulation to include the cases where viscosity depends on temperature. Following their work, the wave speed is taken to be small initially and the disturbances structure analogous to that occurring in two-dimensional boundary layer stability. The linear eigenrelations are derived for profiles with more than one critical layer. However, investigation relating to the asymptotic solutions of a rotating disk with temperature dependent viscosity has not been carried out as yet, which, therefore, is one of the aims of the current work.

This paper is organized as follows: $\S 1$ is devoted to the description of the problem $\S 2$ for basic equation, and asymptotic solution. The conclusions are set in $\S 3$.

\section{The Basic Equations}

We take an infinite disk rotating with uniform angular velocity $\Omega$ about the vertical axis $z$, which passes through the centre of the disk. We consider the three dimensional boundary layer flow of an incompressible fluid. $\mathrm{T}_{\omega}$ denotes the uniform temperature at the disk surface and $T_{\infty}$ is the temperature of the ambient fluid. The basic equations in cylindrical polar coordinates $(\mathrm{r}, \theta, \mathrm{z})$ governing the viscous fluid flow are

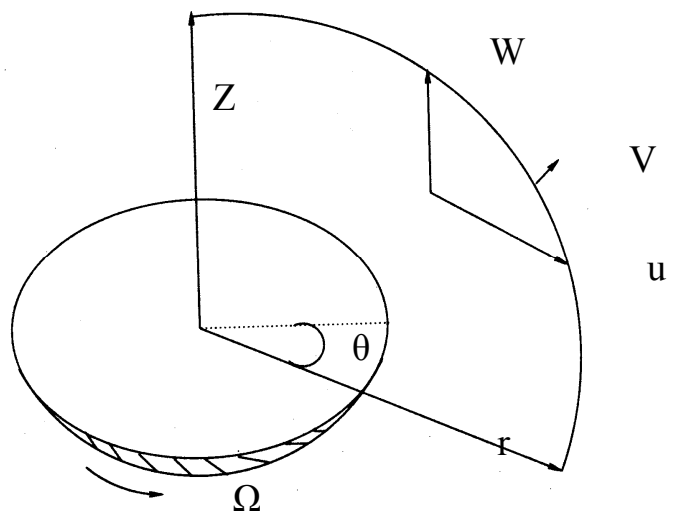

Fig. 1: The flow configuration and coordinate system.

$$
\begin{aligned}
& \rho_{\infty}\left(\frac{\partial U}{\partial t}+(U . \nabla) U\right)=\nabla P+\nabla\left(\mu^{\prime} \nabla U\right), \\
& \nabla . U=0, \\
& \rho_{\infty} C_{P}\left(\frac{\partial T}{\partial t}+(U . \nabla) U\right)=k \nabla^{2} T,
\end{aligned}
$$

where $\mathrm{U}$ is the velocity field with the radial, tangential and vertical components of velocity as $u, v, w$. Here, $C_{p}$ is the specific heat at constant pressure, $\rho_{\infty}$ the fluid density, $k$ the thermal conductivity of the fluid, $p$ the pressure in the flow, and $\mu^{\prime}$ the viscosity of the fluid. In addition, we assume that the viscosity depends on temperature, i.e. $\mu^{\prime}=\mu_{\infty} /[1$ $\left.+\varepsilon\left(T-T_{\infty}\right) /\left(T_{\omega}-T_{\infty}\right)\right]$, where $\in$ is termed the viscosity variation parameter. All other material functions such as the fluid density $\rho$ and the thermal conductivity $k$ of the fluid 
are treated as constants. The Navier-Stokes equations are non-dimensionalized with respect to a length, distance cale $L=r_{\mathrm{e}}^{*}$, velocity scale $U_{c}=L \Omega$, time scale $L / U_{c}$ and pressure scale $\rho_{\infty} U_{\mathrm{c}}^{2}$. This leads to a global Reynolds number $\operatorname{Re}=\frac{U_{c} L}{v}=R^{2}$, where $R$ is the Reynolds number based on the displacement thickness $\delta=\left(\frac{v}{\Omega}\right)^{\frac{1}{2}}$. Thus, relative to non-dimensional cylindrical polar coordinates $(\mathrm{r}, \theta, \mathrm{z})$ which rotate with the disk, the full time-dependent, un-steary Navier-Stokes equations governing the viscous fluid flow are the usual momentum and the continuity equations given as follows

$$
\begin{aligned}
& \frac{\partial u}{\partial t}+u \frac{\partial u}{\partial r}+\frac{u}{r} \frac{\partial u}{\partial \theta}+w \frac{\partial u}{\partial z}-\frac{v^{2}}{2} 2 u-r=\frac{1}{R^{2}}\left[-\frac{1}{r} \frac{\partial P}{\partial r}+\frac{\partial}{\partial r}\left(\mu \frac{\partial u}{\partial r}\right),\right. \\
& \left.+\frac{\partial}{\partial r}\left(\mu \frac{u}{r}\right)+\frac{1}{r 2} \frac{\partial}{\partial \theta}\left(\mu \frac{\partial \mu}{\partial \theta}\right)+\frac{\partial}{\partial z}\left(\mu \frac{\partial \mu}{\partial z}\right)-\frac{2}{r^{2}} \frac{\partial}{\partial \theta}(\mu v)\right], \\
& \frac{\partial u}{\partial t}+u \frac{\partial u}{\partial r}+\frac{u}{r} \frac{\partial u}{\partial \theta}+w \frac{\partial u}{\partial z}+\frac{u v}{r} 2 u=\frac{1}{R^{2}}\left[-\frac{1}{r} \frac{\partial P}{\partial \theta}+\frac{\partial}{\partial r}\left(\mu \frac{\partial u}{\partial r}\right)\right. \\
& \left.+\frac{\partial}{\partial r}\left(\mu \frac{u}{r}\right)+\frac{1}{r 2} \frac{\partial}{\partial \theta}\left(\mu \frac{\partial v}{\partial \theta}\right)+\frac{\partial}{\partial z}\left(\mu \frac{\partial \mu}{\partial z}\right)-\frac{2}{r^{2}} \frac{\partial}{\partial \theta}(\mu v)\right] \\
& \frac{\partial u}{\partial t}+u \frac{\partial u}{\partial r}+\frac{u}{r} \frac{\partial u}{\partial \theta}+w \frac{\partial w}{\partial z}=\frac{1}{R^{2}}\left[-\frac{\partial P}{\partial z}+\frac{\partial}{\partial r}\left(\mu \frac{\partial w}{\partial r}\right)+\frac{1}{2} \frac{\partial}{\partial r}\left(\mu_{t} w\right)\right. \\
& \left.+\frac{1}{r^{2}} \frac{\partial}{\partial \theta}\left(\mu \frac{\partial w}{\partial \theta}\right)+\frac{\partial}{\partial z}\left(\mu \frac{\partial w}{\partial z}\right)\right], \\
& {\left[\frac{\partial T}{\partial t}+u \frac{\partial T}{\partial r}+\frac{u}{r} \frac{\partial T}{\partial z}\right]=\frac{1}{R^{2}} \frac{1}{P_{r}}\left[\frac{\partial^{2} T}{\partial r^{2}}+\frac{1}{r^{2}}\left(\mu \frac{\partial^{2} T}{\partial \theta^{2}}\right)+\frac{1}{r} \frac{\partial T}{\partial r}+\frac{\partial^{2} T}{\partial z^{2}}\right],} \\
& \quad \frac{\partial u}{\partial r}+\frac{u}{r}+\frac{1}{r} \frac{\partial u}{\partial \theta}+\frac{\partial w}{\partial z}=0,
\end{aligned}
$$

where $\mu=1 /[1+\varepsilon(T-T) /(T \omega-T \infty)]$. In this analysis the fluid is assumed to lie in the $z \geq 0$ semi-infinite space. In the above equations, the curvature effects as well as the effects streaming from the Coriolis force are present.

To obtain the solutions of the governing equations, these are first converted into a convenient form using appropriate transformations. The boundary layer coordinate $Z$, which is of order $\mathrm{O}(1)$ is defined as $Z=z R$. Considering this, we can introduce the following mean flow variables:

$$
u_{B}=r F(Z), v_{B}=r G(Z), w=\frac{1}{R} H(Z),
$$




$$
p_{B}=\frac{1}{R^{2}} H(Z), T B=\frac{T-T_{\infty}}{T_{\omega}-T_{\infty}}=S(Z) .
$$

Substituting (3) into (2) yields the following non-dimensional ordinary differential equations for the mean flow:

$$
\begin{aligned}
& (1+\varepsilon S) F^{\prime \prime}-\varepsilon S^{\prime} F^{\prime}-(1+\varepsilon S)^{2}\left[F^{2}-(G+1)^{2}+F^{\prime} H\right]=0, \\
& (1+\varepsilon S) G-\varepsilon S G^{\prime}-\varepsilon S^{\prime} G^{\prime}-(1+\varepsilon S)^{2}\left[2 F(G+1)+G^{\prime} H\right]=0, \\
& S^{\prime \prime}-P_{r} S H=0, \\
& 2 F+H^{\prime}=0,
\end{aligned}
$$

where prime denotes differentiation with respect to $Z$, and $\operatorname{Pr}=\mu_{\infty} C_{p} / \kappa$ is the Prandtl number. The boundary conditions are

$$
\begin{aligned}
& F=0, G=0, H=0, S=1 \text { at } Z=0, \\
& F=0, G=-1, S=0, H=h_{\infty} \text { as } Z \rightarrow \infty .
\end{aligned}
$$

The value of $h_{\infty}$ is a constant vertical velocity of the rotating fluid in the far-field above the disk.

We shall seek the asymptotic solution of mean flow equations (4) for small viscosity variation parameter $\in$. We expand the functions in the ascending power of $\in$ as

$$
\begin{aligned}
& F=F_{0}(Z)+\in F_{1}(Z)+\epsilon^{2} F_{2}(Z)+\ldots, \\
& G=G_{0}(Z)+\in G_{1}(Z)+\epsilon^{2} H_{2}(Z)+\ldots, \\
& H=H_{0}(Z)+\in H_{1}(Z)+\epsilon^{2} H_{2}(Z)+\ldots, \\
& S=S_{0}(Z)+\in S_{1}(Z)+\epsilon^{2} S_{2}(Z)+\ldots,
\end{aligned}
$$

Substituting (6) in equations (4) and equationg the coefficients of $O(1)$, we get the following set of equations

$$
\begin{aligned}
& F_{0}^{\prime \prime}-\left[F_{0}^{2}-\left(G_{0}+1\right)^{2}+F_{0}^{\prime} H_{0}=0,\right. \\
& G_{0}^{\prime \prime}-\left[2 F_{0}\left(G_{0}+1\right)+G_{0}^{\prime} H_{0}\right]=0, \\
& S_{0}^{\prime \prime}-P_{r} S_{0}^{\prime} H_{0}=0, \\
& 2 F_{0}+H_{0}^{\prime}=0 .
\end{aligned}
$$

The corresponding boundary conditions are

$$
\begin{aligned}
& F_{0}=G_{0}=H_{0}=0, S_{0}=1 \text { at } Z=0, \\
& F_{0}=0, G_{0}=-1, S_{0}=0, H_{0}=\text { constant as } Z \rightarrow \infty .
\end{aligned}
$$

Next we equate terms of $O(\in)$ to obtain the equations

$$
\begin{aligned}
& F_{1}^{\prime \prime}+S_{0} F_{0}^{\prime \prime}-\left(S_{0}^{\prime}+H_{1}\right) F_{0}^{\prime}-F_{1}^{\prime} H_{0}+2 G_{1}\left(G_{0}+1\right)-2 F_{0} F_{1} \\
& -2 S_{0}\left[F_{0}^{2}-\left(G_{0}+1\right)^{2}+F_{0}^{\prime} H_{0}\right]=0,
\end{aligned}
$$




$$
\begin{aligned}
& \left.G_{1}^{\prime \prime}+S_{0} G_{0}^{\prime \prime}-\left(S_{0}^{\prime}+H_{1}\right) G_{0}^{\prime}-G_{1}^{\prime} H_{0}-2 F_{1}\left(G_{0}+1\right)-2 F_{0}\left(G_{0}-1\right)+G_{0}^{\prime} H_{0}\right]=0, \\
& S_{1}^{\prime \prime}-P_{r}\left(S_{0}^{\prime} H_{1}+S_{0}^{\prime} H_{0}\right)=0, \\
& 2 F_{1}+P_{r} H_{1}^{\prime}=0 .
\end{aligned}
$$
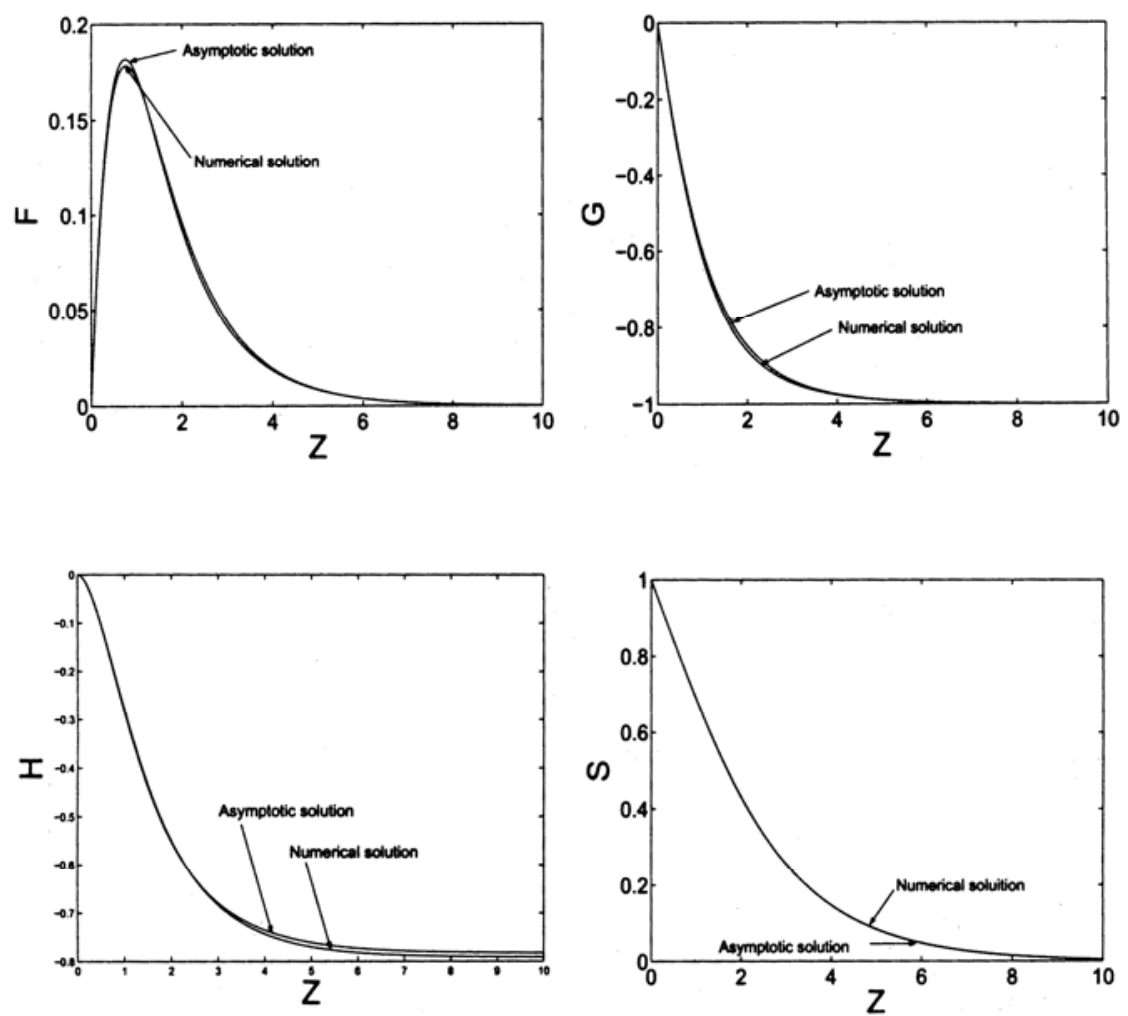

Fig. 2: A comparison of the numerical and asymptotic calculations of mean velocity Profile (a) radial $F$, (b) azimuthal $G$, (c) axial $H$, and (d) temperature profile $S$ as a function of $Z$ when $\varepsilon=0.05$.

The corresponding boundary conditions are

$$
\begin{aligned}
& F_{1}=G_{1}=H_{1}=S_{1}=0 \text { at } Z=0, \\
& F_{1}=G_{1}=S_{1}=H_{1}=0 \text { as } Z \rightarrow \infty .
\end{aligned}
$$

We have solved the set of ordinary differential equations (7) and (9) with the appropriate boundary conditions by employing the finite difference method for $F_{i}, G_{i}, H_{i}$, and $\mathrm{S}_{i}(i=$ 0 , and 1).

Based on these asymptotic findings, comparisons with the numerical calculations are sketched in figures (2) for temperature-dependent viscosity parameter $\varepsilon=0.05$. Graphs show that the asymptotic solution is consistent with numerical computation fives by [9] for small temperature-dependent viscosity parameter $\varepsilon$. 


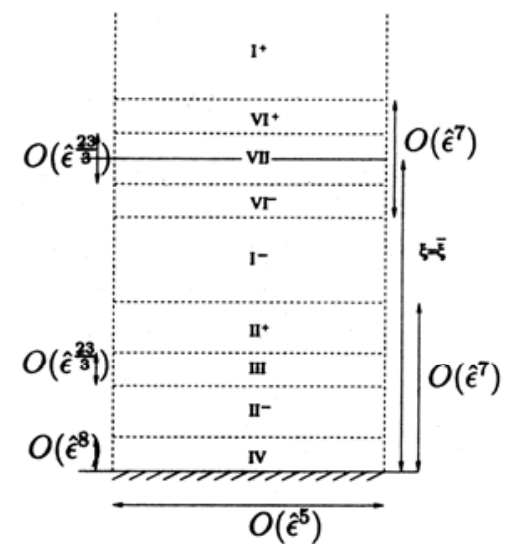

Fig. 3: Flow structure for infinitesimal sized disturbances.

We are here interested in perturbation solution of the basic velocity profiles using liear theory. A small parameter $\hat{\varepsilon}=R-\frac{1}{12}$ is defined. The scalings and disturbances structure with an infinitesimal sized perturbation having wave speed $O(\hat{\varepsilon})$ is imposed onto the basic flow solution. Following the scalings and basic structure given by Bassom \& Gajjar [10], a critical layer for positive wave speeds is determined close to the disk surface. In figure 3, we have displayed the zone structure near the disk surface. Most of the boundary layer is contained in zone I whereas zone II constitutes inviscid adjustment housing thinner zones III and IV which characterize the critical layer and viscous wall layer, respectively. The zone II is bifurcated into two regions by layer III designated as II ${ }^{-}$and $\mathrm{II}^{+}$in figure 3. Zones VII are embeded in zone I. We consider the thicknesses of zones IIV as $O\left(\hat{\varepsilon}^{6}\right), O\left(\hat{\varepsilon}^{7}\right), O\left(\hat{\varepsilon}^{23 / 3}\right)$, and $O\left(\hat{\varepsilon}^{8}\right)$, and streamwise lengthscale $O\left(R^{-5 / 12}\right)$. The basic flow is perturbed as

$$
(u, v, w, T, P)=\left[\left(u_{B}, v_{B}, w_{B}, T_{B}, p_{B}, p_{B}\right)+\delta(U, V, W, T, P)\right]+\ldots .,(\delta<<1)
$$

where $u, v$, and $\mathrm{w}$ are velocity components, $\mathrm{T}$ is the temperture profile, and $\mathrm{p}$ is the pressure.

First, we consider zone I, which forms the majority of the boundary layer. We define $z=$ $\hat{\varepsilon}^{6} \xi$ and $\xi=O(1)$, and expand $U, V, W, T$, and $P$ in the form

$$
(U, V, W, T, P)=\left[\left(u_{0}, \mathrm{v}_{0}, w_{0}, T_{0}, p_{0}\right)+\hat{\varepsilon}\left(u_{1}, \mathrm{v}_{1}, w_{1}, T_{1}, p_{1}\right)+\ldots .\right] E+\text { c.c. }
$$

where $E=\exp (i(\bar{x}(r, \theta, t)))$ and c.c denotes the complex conjugate. Applying the method of multiple scales, we obtain

$$
\begin{aligned}
& \frac{\partial E}{\partial r}=i\left(\frac{\alpha_{0}}{\hat{\varepsilon}^{5}}+\frac{\alpha_{0}}{\hat{\varepsilon}^{4}}+\ldots\right) E+\frac{\partial E}{\partial r}, \\
& \frac{\partial E}{\partial r}=i\left(\frac{\beta_{0}}{\hat{\varepsilon}^{5}}+\frac{\beta_{1}}{\hat{\varepsilon}^{4}}+\ldots\right) E,
\end{aligned}
$$




$$
\frac{\partial E}{\partial t}=i\left(\frac{\alpha_{0}}{\hat{\varepsilon}^{5}}+\frac{\alpha_{1}}{\hat{\varepsilon}^{4}}+\ldots\right)\left(c_{0} \hat{\varepsilon}+c_{1} \hat{\varepsilon}^{2}+\ldots .\right) E
$$

where wavenumbers $\alpha_{j}, \beta_{j}$, and frequency $c_{j}(j=0,1,2, \ldots)$ are taken to be real. These expansions are then substituted into $N-S$ equation (2) and the leading order terms are equated. The solution of the system is

$$
u_{0}=A F^{\prime}, u_{0}=A G^{\prime}, w_{0}=-(i A / r) U_{0 B}, T_{0}=A S^{\prime}, p_{0}=P c,
$$

where $A$ and $P_{c}$ are unknown constants and where we have defined $U_{n B}=r \alpha_{n} F(\xi)$, + $\beta_{n} G(\xi), n=0,1,2, \ldots$. .Equating the next order terms and performing some manipulations, we find that

$$
w_{1}=-\frac{i A}{r}\left(U_{1 B}-\alpha_{0} C_{0}\right)+i \gamma_{0}^{2} P_{c} U_{0 B} \int^{\xi} \frac{d \xi}{\left(U_{0 B}\right)^{2}}+i A_{1} U_{0 B},
$$

where $A_{1}$ is also an unknown constant and leading-order wavenumber $\gamma_{0}^{2}=\alpha_{0}^{2}+\beta_{0}^{2} r^{-2}$. We write $\mu=\mu_{0}+\varepsilon \mu_{1}+\ldots, \alpha_{0}=\hat{\alpha}_{0}+\varepsilon \hat{\alpha}_{1}+\ldots$, and $\beta_{0}=\hat{\beta}_{0}+\varepsilon \hat{\beta}_{1}+\ldots$, where $\varepsilon$ is the temperature-dependent viscosity parameter assumed to be small. If there exists a non-zero $\xi=\bar{\xi}$ (upper critical layer position) such that $U_{0 B}(\bar{\xi})=0$, then there is a critical layer in zone I distinct form the critical layer at the wall. If $\mu_{0}=\hat{\beta}_{0} / r \hat{\alpha}_{0}$, and $\mu_{1}=\hat{\beta}_{1} / r \hat{\alpha}_{0}-\hat{\beta}_{1} / r \hat{\alpha}_{0}-\hat{\beta}_{0} \hat{\alpha}_{1} / \hat{\alpha}_{0}^{2}$, then is given by the condition

$$
\left.U_{0 B}\right|_{(\xi-\bar{\xi})}=0 \text {, or }\left.(F+\mu G)\right|_{(\xi=\bar{\xi})}=0 \text {. }
$$

$\bar{\xi}$ exists if $0<\mu<\mu_{\mathrm{c}}$ where

$$
\mu_{\mathrm{c}}=-\left(\frac{F_{0}^{\prime}}{G_{0}^{\prime}}\right)_{\xi=0}-\left.\left(\frac{\left(F_{1} G_{0}^{\prime}+F_{0}^{\prime} G_{1}^{\prime}\right)}{\left(G_{0}^{\prime}\right)^{2}}\right)\right|_{\xi=0} .
$$
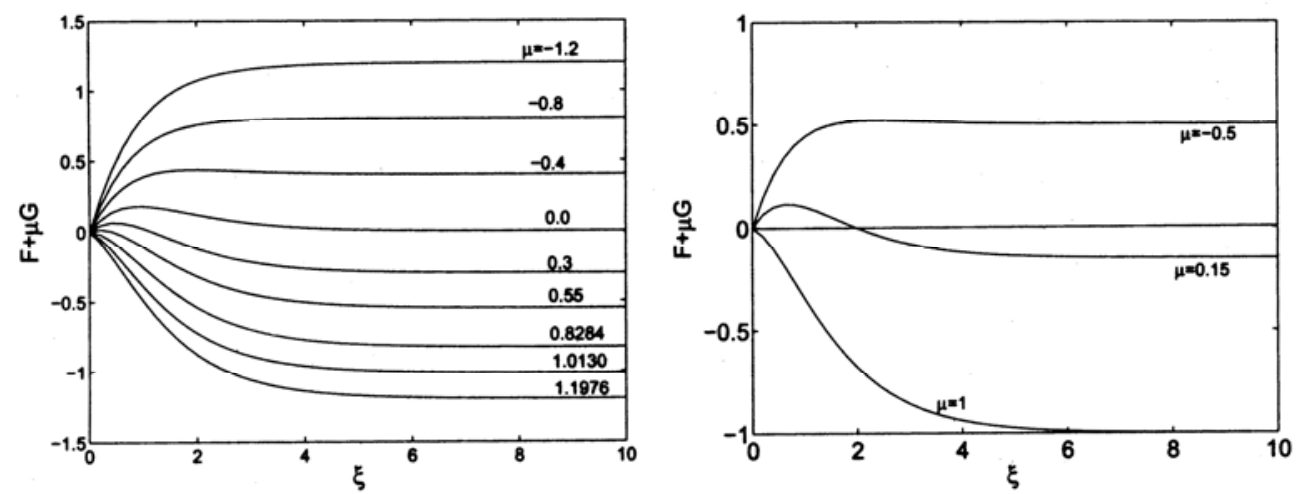

Fig. 4: Mean velocity profile $(F+\mu G)$ as a function of $\xi$ for different values of $\mu$.

When $\varepsilon=0$, the critical value of $\mu$ is $\mu_{c}=-\left(\frac{F_{0}^{\prime}}{G_{0}^{\prime}}\right)_{\xi=0} \approx 0.8284$, 
which is the same value as obtained by Bassom \& Gajjar [10].

When $\varepsilon=0.05,0.1,0.2,0.3$, and 0.4 , the critical vales of $\mu_{\mathrm{c}}$ are $0.8745,0.9207,1.0130$, and 1.1976, respectively. Figure 4 shows that $\bar{\xi}$ exists if $0<\mu<\mu_{c}$.

The flow structure is augmented by zones VI and VII (see figure 3) of thicknesses $O\left(\hat{\varepsilon}^{7}\right)$ and $O\left(\hat{\varepsilon}^{23 / 3}\right)$ centered on $\xi=\bar{\xi}$, when the second critical layer exists at some point in zone $I$.

In zone II, $z=\hat{\varepsilon}^{7} Z=O(1)$. The perturbation quantities take form

$$
\left.(U, V, W, T, P)=\left[\left(u^{0}, v^{0}, \hat{\varepsilon}^{2} w^{0}, T^{0}, \hat{\varepsilon} p^{0}\right)+\hat{\varepsilon}\left(u^{1}, v^{1}, \hat{\varepsilon}^{2} w^{1}, T^{1}, P^{1}\right)+\ldots . .\right] E+c . c\right]
$$

The above expansions are then substituted into N-S equation (2) and the leading-order terms are equated. We obtain the solutions for $Z>Z_{c}$. where $Z_{c}$ denotes the lower critical point equal to $\alpha_{0} \mathrm{c}_{0} / \lambda_{10}$ :

$$
\begin{aligned}
u^{0} & =\frac{-\alpha_{0} P c+A\left(F^{\prime}\right)_{\xi=0} \lambda_{10} Z}{\left(\lambda_{10} Z-\alpha_{0} c_{0}\right)}, u^{0}=\frac{-\beta_{0} P_{c}+A\left(G^{\prime}\right)_{\xi=0} \lambda_{10} Z}{r\left(\lambda_{10} Z-\alpha_{0} c_{0}\right)}, \\
T^{0} & =\frac{A\left(S^{\prime}\right)_{\xi=0} \lambda_{10} Z}{\left(\lambda_{10} Z-\alpha_{0} c_{0}\right)}, P_{0}=P_{c}, \\
w^{0} & =-\frac{i \mathrm{~A} \lambda_{10} Z}{\mathrm{r}}+i\left(\frac{\alpha_{0} c_{0} A}{r}-\frac{\gamma_{1}^{2} P_{c}}{\lambda_{10}}\right),
\end{aligned}
$$

where we have defined

$$
\lambda_{\mathrm{n} 0}=\lambda_{n 0}=\left.\left(\frac{d^{n} U_{0 B}}{d \xi^{n}}\right)\right|_{\xi=0}
$$

For the inviscid constraint which requires $w(0) \rightarrow 0$ as $Z \rightarrow 0$, equations (14c) furnish

$$
\alpha_{0} c_{0} A \lambda_{10}=r \gamma_{0}^{2} P_{c} .
$$

For the next order, the solution given is

$$
\begin{aligned}
& \left.\left.w^{(1)}=\frac{i A \lambda_{20}}{2 r}\left(Z-Z_{c}\right)^{2}+i A^{(1)} \lambda_{10}\right) Z-Z_{c}\right) \\
& -\frac{i A_{0} \alpha_{0} c_{0} \lambda_{20}}{r \lambda_{20}}\left(Z-Z_{c}\right)\left[\operatorname{In}\left(Z-Z_{e}\right)+\operatorname{In}|\lambda 10|\right]+i A_{2},
\end{aligned}
$$

where $A_{2}$ is a real constant. This solution is valid only for $Z>Z_{c}$. Details of the critical layer at $Z=Z_{c}$ are now required for the continuation below $Z<Z_{c}$. Following Bassom \& Gajjar [10], equation (16) holds for $Z<Z_{c}$ if $\operatorname{In}\left(Z-Z_{c}\right)$ is replaced by [In $\left.\left(Z-Z_{c}\right)-i \pi\right]$ when $\lambda_{10}>0$ pr bu $\left[\operatorname{In}\left(Z-Z_{c}\right)+i \pi\right]$ when $\lambda_{10}<0$. 
Zone IV, describes the viscous wall layer, where $z=\hat{\varepsilon}^{8} \hat{z}$ and $\hat{z}=O(1)$. The perturbation quantities now take the form

$$
(u, v, w, T, p)=\left[\left(\hat{u}_{0}, \hat{\mathrm{v}}_{0}, \hat{\varepsilon}^{3} \hat{w}_{0}, \hat{T}_{0}, \hat{\varepsilon} \hat{p}_{0}\right)+\ldots\right] E+\text { c.c. }
$$

For $\alpha_{0} c_{0}>0$, the solutions derived are

$$
\begin{aligned}
& \hat{u}_{0}=\frac{P_{c}}{C_{0}}(1-\exp (-k \hat{z})), \quad \hat{v}_{0}=\frac{\beta_{0} P_{c}}{\alpha_{0} c_{0}}(1-\exp (-k \hat{z})), \\
& \hat{T}_{0}=-\exp \left(-k P_{r} \hat{z}\right), \quad \hat{w}_{0}=\frac{i \lambda_{10} A_{0}}{r m}(k \hat{z}-1-\exp (-k \hat{z})),
\end{aligned}
$$

where $k=e^{-\frac{1}{4} i \pi} \sqrt{\left(\alpha_{0} c_{0}\right)}$ and $P_{r}$ is the Prandtl number.

In zone $V, z=\hat{\varepsilon}^{5} \bar{z}$ and $\bar{z}=O(1)$. Thickness $O\left(\hat{\varepsilon}^{5}\right)$ is therefore required to describe the flow in the far-field. The perturbation quantities can be expresed as

$$
(u, \mathcal{v}, w, T, p)=\left[\hat{\varepsilon}\left(\hat{u}_{0}, \hat{\mathrm{v}}_{0}, \hat{w}_{0}, \hat{T}_{0}, \hat{p}_{0}\right)+\ldots\right] E+\text { c.c. }
$$

For large $\xi, F \rightarrow O(\exp (-c \xi)), G \rightarrow-1+O(\exp (-c \xi))$ where $c=0.884$. Hence, solutions in this zone are

$$
\begin{aligned}
& \hat{u}_{0}=\frac{\alpha_{0} P_{c}}{\beta_{0}}\left(\exp \left(-\gamma_{0} \bar{z}\right)\right), \quad \bar{v}_{0}=\frac{P_{c}}{r}\left(\exp \left(-\gamma_{0} \bar{z}\right)\right), \\
& \bar{w}_{0}=\frac{i_{0} P_{c}}{\beta_{0}}\left(\exp \left(-\gamma_{0} \bar{z}\right)\right), \quad \bar{p}_{0}=P_{c}\left(\exp \left(-\gamma_{0} \bar{z}\right)\right), \\
& \bar{T}_{0}=\frac{r \gamma_{0} S^{\prime} P_{c}}{\beta_{0}}\left(\exp \left(-\gamma_{0} \bar{z}\right)\right) .
\end{aligned}
$$

Matching with zone I requires therefore,

$$
\lambda_{10} \alpha_{0} c_{0}=\gamma_{0} \beta_{0}^{2}
$$

There are two critical layers in the flow when $\alpha_{0}$ and $c_{0}$ are both positive. There is a phase jump of $\pi$ in $w_{1}$ across the upper critical layer. if

$$
w_{1}=\frac{i A}{r}\left(U_{1 b}-\alpha_{0} c_{0}\right)+i \gamma_{0}^{2} P_{c} U_{0 B} \int^{\xi} \frac{d \xi}{\left(U_{0 B}\right)^{2}}+i A_{1} U_{o B},
$$

with $A, A_{1}$ and $P_{0}$ as real for $\xi>\bar{\xi}$, then $\xi>\bar{\xi}$, and $A_{1}$ needs to be replaced by

$$
A_{1}^{*}=(\text { real quantity })-\frac{i \pi \lambda_{2 \bar{\xi}} \lambda_{0}^{2} P^{(0)}}{\left(\lambda_{1 \bar{\xi}}\right)^{3}},
$$


where $\lambda_{n 0}=\left.\left(\frac{d^{n} U_{0 B}}{d \xi^{n}}\right)\right|_{\xi=\bar{\xi}}$. This replacement yields the solution valid for $\xi>\bar{\xi}$.

When two critical layers occur, matching the velocities between zones $\mathrm{II}^{-}$and $\mathrm{IV}$, the resulting equations are

$$
\begin{aligned}
& \mu=\beta_{d} / \alpha_{d}, \\
& 2^{\frac{1}{2}} \pi \alpha_{d}^{\frac{1}{2}} c_{d}^{\frac{5}{2}} x=-1 \\
& X=\left[-\frac{\left(F^{\prime \prime}+\mu G^{\prime \prime}\right)_{\xi=\bar{\xi}}}{\left(F^{\prime}+\mu G^{\prime}\right)_{\xi=\bar{\xi}}^{3}}-\frac{1}{\left(F^{\prime}+\mu G^{\prime}\right)_{\xi=0}^{3}}\right], \\
& c_{d}\left(F^{\prime}+\mu G^{\prime}\right)_{\xi=0}=\alpha_{d} \mu^{2}\left(1+\mu^{2}\right)^{\frac{1}{2}},
\end{aligned}
$$

where $\alpha_{d}=r^{\frac{1}{2}} \alpha_{0}, \quad \beta_{d}=r^{-\frac{5}{6}} \beta_{0}, \quad c_{d}=r^{-\frac{5}{6}} c_{0}$. Here $\bar{\xi}$ denotes the upper critical layer position.

We shall extend the above formulation for temperature-dependent viscosity parameter $\varepsilon$, where $\varepsilon$ is small. We expand $\alpha_{d}, \beta_{d}, c_{d}, X$, and $\mu$ in the ascending power of $\varepsilon$ as

$$
\begin{aligned}
& \alpha_{d}=\hat{\alpha}_{0}+\varepsilon \hat{\alpha}_{1}+\varepsilon^{2} \hat{\alpha}_{2}+\ldots, \\
& \beta_{d}=\hat{\beta}_{0}+\varepsilon \hat{\beta}_{1}+\varepsilon^{2} \hat{\beta}_{2}+\ldots, \\
& c_{d}=\hat{c}_{0}+\varepsilon \hat{c}_{1}+\varepsilon^{2} \hat{c}_{2}+\ldots, \\
& \mu=\mu_{0}+\varepsilon \mu_{1}+\varepsilon^{2} \mu_{2}+\ldots, \\
& X=X_{0}+\varepsilon X_{1}+\varepsilon^{2} X_{2}+\ldots,
\end{aligned}
$$

Substituting (23) into equations (22) and equating the terms of $O(1)$, the following set of equations:

$$
\begin{aligned}
& \mu_{0}=\hat{\beta}_{0} / \hat{\alpha}_{0}, \\
& 2^{\frac{1}{2}} \pi \hat{\alpha}_{0}^{\frac{1}{2}} \hat{c}_{0}^{\frac{5}{2}} X_{0}=-1 \text {, } \\
& X_{0}=\left[-\left[\begin{array}{c}
\left(F_{0}^{\prime \prime}+\mu_{0} G_{0}^{\prime \prime}\right)_{\xi=\bar{\xi}} \\
{\left[\left(F_{0}^{\prime}+\mu_{0} G_{0}^{\prime}\right)_{\xi=\bar{\xi}}\right.}
\end{array}\right]-\left[\begin{array}{c}
1 \\
{\left[\left(F_{0}^{\prime}+\mu_{0} G_{0}^{\prime}\right)^{3}\right]_{\xi=\bar{\xi}}}
\end{array},\right.\right. \\
& \hat{c}_{0}\left(F_{0}^{\prime}+\mu_{0} G_{0}^{\prime}\right)_{\xi=0}=\hat{\alpha}_{0} \mu_{0}^{2}\left(1+\mu_{0}^{2}\right)^{\frac{1}{2}} \text {. }
\end{aligned}
$$


Next, we equate terms of $O(\varepsilon)$ to get

$$
\begin{aligned}
& \mu_{1}=\left[\frac{\hat{\beta}_{1}}{\hat{\alpha}_{0}}-\frac{\hat{\beta}_{0} \hat{\alpha}_{0}}{\hat{\alpha}_{0}^{2}}\right], \\
& X_{1}=\frac{\left(F_{1}^{\prime \prime}+\mu_{0} G_{1}^{\prime \prime}+G_{0}^{\prime \prime}\right)_{\xi=\bar{\xi}}}{\left[\left(F_{0}^{\prime}+\mu_{0} G_{0}^{\prime}\right)^{3}\right]_{\xi=\bar{\xi}}}+\frac{3\left(F_{1}^{\prime}+\mu_{0} G_{1}^{\prime}+\mu_{1} G_{0}^{\prime}\right)_{\xi=0}}{\left[\left(F_{0}^{\prime}+\mu_{0} G_{0}^{\prime}\right)^{4}\right]_{\xi=0}}+\frac{3\left(F_{0}^{\prime \prime}+\mu_{0} G_{0}^{\prime \prime}\right)\left(F_{1}^{\prime \prime}+\mu_{0} G_{1}^{\prime \prime}+\mu_{1} G_{0}^{\prime \prime}\right)}{\left[\left(F_{0}^{\prime}+\mu_{0} G_{0}^{\prime}\right)^{4}\right]_{\xi=0}}
\end{aligned}
$$

$$
\begin{aligned}
& \hat{\alpha}_{1}=-\frac{(5 / 3)\left(F_{1}^{\prime}+\mu_{0} G_{1}^{\prime}+\mu_{1} G_{0}^{\prime}\right)_{\xi=0}\left[\left(F_{0}^{\prime}+\mu_{0} G_{0}^{\prime}\right)_{\xi=0}^{3 / 2}\right.}{2^{3 / 2} \mu_{0}^{5}\left(1+\mu_{0}^{2}\right)^{5 / 4} \chi_{0} \hat{\alpha}_{0}^{2}}, \\
& -\frac{\hat{\alpha}_{0}}{3 \chi_{0}}\left(\chi_{1}+\frac{5 \mu_{1} X_{0}}{\mu_{0}}+\frac{5 X_{0} \mu_{0} \mu_{1}}{1+\mu_{0}^{2}}\right)
\end{aligned}
$$$$
\hat{c}_{1}=\frac{\mu_{0}^{2}\left(1+\mu_{0}^{2}\right)^{\frac{1}{2}}}{\left(F_{0}^{\prime}+\mu_{0} G_{0}^{\prime}\right)_{\xi=0}}\left[\hat{\alpha}_{1}+\hat{\alpha}_{0}\left(\frac{2 \mu_{1}}{\mu_{0}}+\frac{\mu_{0} \mu_{1}}{1+\mu_{0}^{2}}-\frac{\hat{\alpha}_{0}\left(F_{0}^{\prime}+\mu_{0} G_{1}^{\prime}+\mu_{1} G_{0}^{\prime}\right)_{\xi=0}}{\left(F_{0}^{\prime}+\mu_{0} G_{1}^{\prime}\right)_{\xi=0}}\right)\right] .
$$
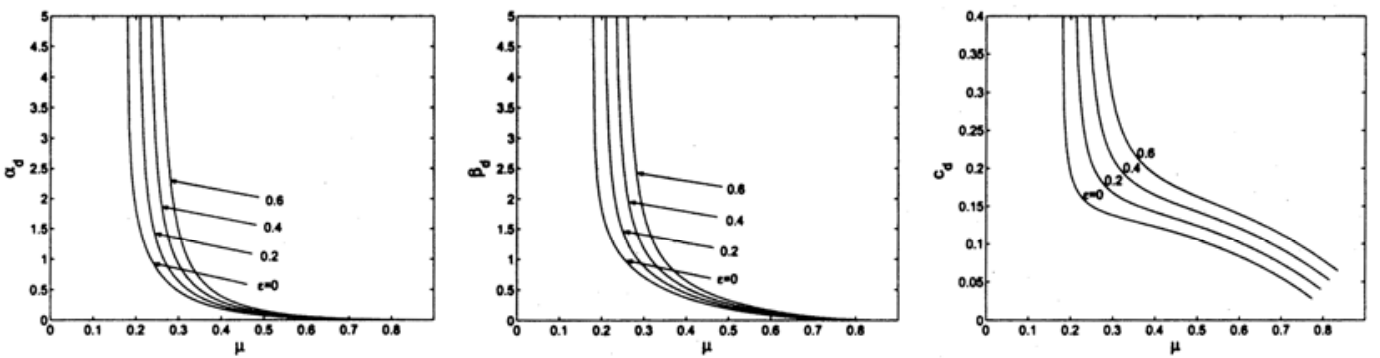

Fig. 5: (a) Wavenumber $\alpha_{d}$, and (b) wavenumber $\beta_{d}$ as a function of $\mu$, and (c) wave speed $c_{d}$ as a function of $\mu$ for different values of temperature-dependent viscosity $\varepsilon$.
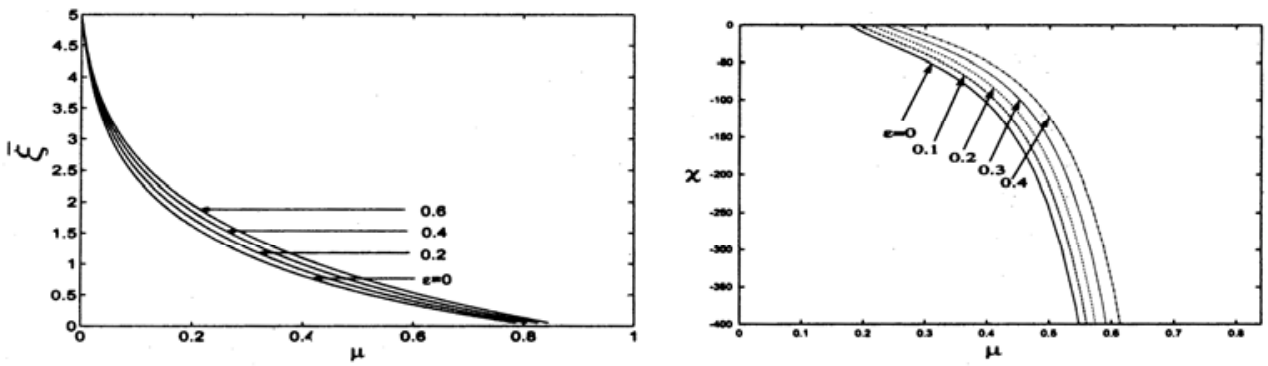

Fig. 6: (a) $\bar{\xi}$, and (b) $X$ as a function of $\mu$ for different values of temperature-dependent viscosity $\varepsilon$.

We have determined wavenumbers, wave speed, $\bar{\xi}$ and $X$ from equations (24) and (25). These solutions are sketched in figures 5 and figures 6 for different values of temperature dependent viscosity. These figures show that solutions exist only for $\mu_{1 c}<\mu<\mu_{c}$ and $\mu<$ 0 , where $\mu_{1 c} \approx 0.183,0.189,0.206,0.232$, and 0.253 for $\varepsilon=0,0.05,0.1,0.2,0.3$ and 0.4 , respectively, and $\mu_{c} \approx 0.8284,0.8745,0.9207,1.0130$ and 1.1976 for $\varepsilon=0,0.05,0.1,0.2$, 
0.3 and 0.4 , respectively. For $\mu>\mu_{c}$, the critical layer in Zone $I$ is no longer present and no solutions of eigenrelations (24) and (25) exist. As temperature dependent viscosity parameter $\varepsilon$ is increased, the critical value of $\mu_{c}$ increases. Moreover, $X$ is positive for $0<$ $\mu<\mu_{1 c}$ and $\varepsilon<0.8$. For this range of $\mu$ and $\varepsilon$, there can be no solution with $\alpha_{0}$ and $c_{0}$.

\section{CONCLUSION}

The wave disturbances we have considered make the waveangle $\arctan (\mu)$ with the radius of the disk. As temperature dependent viscosity parameter $\varepsilon$ is increased, the range of waveangle increases. The values $\mu_{1 c}$ waveangle for stationary mode and $\mu_{c}$ for zero shear stress represent cut-off values. The change of $\varepsilon$ has most effect on shear stress mode. It has been found that there is a cut-off value at an angle that lies between $10.3^{\circ}$ and $57.4^{\circ}$, so that solutions exist only for this range. Our results agree quite well with those derived earlier by Bassom \& Gajjar [10] for temperature dependent viscosity parameter $\varepsilon=0$.

\section{REFERENCES}

1. J. Gary, D. R. Kassory, H. Tadjeran, A. Zebib, The effect of significant viscosity variation on convective heat transport in water-saturated porous media, J. Fluid Mech. 117 (1982) 233-249.

2. K. N. Mehta, S. Sood, Transient free convection flow with temperature dependent viscosity in fluid saturated porous medium, Int. J. Eng. Sci. 30 (1992) 1083-1087.

3. N. G. Kafoussias, E. W. Williams, The effect of temperature dependent viscosity on the free convective laminar boundary layer flow past a vertical isothermal flat plate, Acta Mech. 110 (1995) 123-137.

4. N. G. Kafoussias, D. A. S. Rees, Numerical studies of the combined free and forced convective laminar boundary layer flow past a vertical isothermal flat plate with temperature dependent viscosity, Acta Mech. 127 (1998) 39-50.

5. H. B. Keller, Numerical methods in the boundary layer theory, Annual. Rev. Mechanics. 10 (1978) 417433.

6. M. A. Hossain, A. Hossain, M. Wilson, Unsteady flow of viscous incompressible fluid with temperaturedependent viscosity due to a rotating disc in presence of transverse magnetic field and heat transfer, Int. J. Therm. Sci. 40 (2001) 11-20.

7. M. A. Hossain, S. Kabir, D. A. S. Rees, Natural convection of fluid with variable viscosity from a heated vertical wavy surface, Z. Angew. Math. Phys. 53 (2002) 48-57.

8. S. Ferro, G. Gnavi, Effects of temperature-dependent viscosity in channels with porous walls, Phys. Fluids. 14 (2002) 829-849.

9. H. A. Jasmine, J. S. Gajjar, Absolute and convective instabilities in the incompressible boundary layer on a rotating disk with temperature-dependent viscosity, Int. J. Heat and Mass Trans. 48 (2005) 1022-1037.

10. A. P. Bassom, J. S. B. Gajjar, Non-stationary crossflow vortices in a three dimensional boundary layer, Proc. Roy. Soc. London Ser. A 417 (1988) 179-212. 